\title{
ICER report on Alzheimer's disease: implications from a patient perspective
}

\author{
Meg Franklin, PharmD, PhD
}

Alzheimer's disease (AD) affects nearly 6 million people in the United States and is expected to affect more than 14 million people by 2060., ${ }^{1,2}$ The cost of caring for patients with AD was estimated to be $\$ 305$ billion in 2020 and is expected to increase to more than \$1 trillion as the population ages. ${ }^{1}$ On June 7, 2021, the US Food and Drug Administration (FDA) approved Aduhelm (aducanumab) for the treatment of $\mathrm{AD}$ with mild cognitive impairment or mild dementia stage of disease through the accelerated approval pathway based on the reduction of amyloid beta plaques observed in patients treated with the drug.,4 Aducanumab is administered as an intravenous infusion every 4 weeks, at a cost of $\$ 56,000$ per year. ${ }^{4}$ The Institute for Clinical and Economic Review (ICER) recently conducted an assessment of aducanumab for AD. ${ }^{5}$ This commentary focuses on the findings of the assessment and policy recommendations and their implications from a patient perspective.

\section{ICER Report and Recommendations}

The ICER assessment was conducted from a payer and a societal perspective and used the 2 identical phase 3 randomized clinical trials conducted by Biogen-ENGAGE and EMERGE. The ICER review committee unanimously found the evidence to be insufficient to determine the net health benefit of aducanumab, given the uncertainty of benefits, and certainty that harms can occur in patients treated with aducanumab. ${ }^{5}$ The annual health-benefit price benchmark as determined by ICER ranged from $\$ 3,000$ to $\$ 8,400$, depending on the threshold and perspective, which subsequently means that an $85 \%-95 \%$ discount from the listed $\$ 56,000$ annual price would be required to reach the threshold price as set by the assessment. ${ }^{5}$

Several mitigating factors precluded a straightforward assessment of aducanumab. Both clinical trials randomized patients with early $\mathrm{AD}$ to low- or high-dose aducanumab or placebo, and the primary clinical outcome was change in the mean score on the Clinical Dementia Rating ScaleSum of Boxes (CDR-SB). Aducanumab effectively removed beta amyloid at all doses, but both trials were terminated following a prespecified interim analysis for futility. A potential positive treatment effect was noted in the EMERGE trial, although the definition of improvement on the CDR-SB has not been solidified. Results from the ENGAGE trial failed to yield any improvement on the CDR-SB in the high-dose group when compared with placebo. $^{5}$

Further confounding matters was the FDA's role in the regulatory process. As stated by ICER in its policy recommendations, the FDA should

\section{Author affiliation}

Meg Franklin, PharmD, PhD, Franklin Pharmaceutical Consulting, LLC, Rock Hill, SC.

AUTHOR CORRESPONDENCE:

Meg Franklin,

mfranklin@franklin-pharma.com

J Manag Care Spec Pharm 2021;27(11):1618-20

Copyright $@ 2021$, Academy of Managed Care Pharmacy. All rights reserved.

"set a clearer regulatory framework in place by specifying a threshold range for amyloid clearance that will be accepted going forward as 'reasonably likely' to provide patient benefit."' The FDA advisory panel that was convened voted against approval, but further deliberation by the FDA resulted in approval, not on the basis of the interpretation of the clinical outcomes data from the trials, but by pivoting and considering amyloid clearance to be a surrogate endpoint now considered "reasonably likely" to lead to a benefit. This shift was controversial since no data were ever disclosed to show the correlation of amyloid clearance with cognitive outcomes from the clinical trials, and the accelerated approval pathway was leveraged to approve 
a drug in a therapeutic area where clinical outcomes measures do exist and can be measured in relatively short clinical trials. ${ }^{6}$

In its assessment, ICER pooled the results of the 2 trials, which under ordinary circumstances may have raised concern from a methodological perspective, but given the existing complexities underlying the trials and the interpretation of the results, this likely did not affect the final conclusions drawn by the committee. Furthermore, ICER performed sensitivity analyses and did a thorough examination of all of the evidence presented from the trials.

The cost-effectiveness of aducanumab in addition to supportive care was evaluated against supportive care alone. From a health care system perspective, the percentage of patients that could be treated before crossing the ICER potential budget impact threshold of $\$ 819$ million per year is $2.5 \%$ (approximately 35,000 of the 1.4 million AD patients eligible for treatment with aducanumab). These results prompted ICER to issue an access and affordability alert for aducanumab. ${ }^{5}$

\section{Implications for Patients with AD}

\section{HEALTH DISPARITIES}

Health disparities exist in the US health care system, and patients with $\mathrm{AD}$ are no exception. Black and Hispanic patients are 1.5 to 2 times more likely to develop $\mathrm{AD}$, yet in the ENGAGE and EMERGE trials, the patient population was remarkably homogenous, since $0.6 \%$ of patients were Black and $1.5 \%$ were Hispanic. ${ }^{5}$ ICER faced some criticism from the public comments for using this against aducanumab, but the population of the trials only serves to highlight the disparities in treatments. The American Academy of Neurology (AAN) also echoed ICER's concerns regarding the lack of racial and ethnic diversity in the clinical trial population, given the disproportionate impact of $\mathrm{AD}$ on Black and Hispanic patients.

It can be surmised that the lack of diversity in the trial populations is indeed problematic, since there are potential biomarker implications in AD that may vary by ethnicity. Evidence of the magnitude of the variance is still not well defined but appears to be a consideration that could carry ramifications for different populations. ${ }^{7}$ Understanding amyloid clearance with aducanumab and the relationship to ethnicity could also be important from a clinical and safety perspective. ENGAGE and EMERGE combined had approximately $35 \%$ of patients who experienced amyloidrelated imaging abnormalities (ARIA) that ranged in severity from asymptomatic to having to discontinue treatment. ${ }^{5}$ Because the trial populations were predominately White, any differences in safety attributable to ethnicity were unlikely detected.

\section{APPROPRIATE USE AND EXPECTATIONS FROM ADUCANUMAB TREATMENT}

The population studied in the trials was one of mild cognitive impairment or mild dementia. Initially, the FDA issued blanket approval for the drug but later narrowed the scope of the approved label. The Alzheimer's Association has shown support for the FDA's narrowing of the label. ${ }^{8}$ ICER stated in its policy recommendations that, in order to keep patients and their families from being misled, a collaborative effort should be employed to characterize potential benefits of aducanumab for slowing the natural progression of the disease, and not as a cure or a treatment resulting in "improvement" or "return to quality of life." Given the lack of treatment options for patients with $\mathrm{AD}$, patients and caregivers are desperate for a viable option, and setting realistic expectations and ensuring that patients and caregivers are grounded in the limitations and expectations from treatment with aducanumab is paramount. Some providers have expressed concern regarding the approval and use of aducanumab, and several prominent medical centers have announced that they will not currently recommend aducanumab. ${ }^{9-11}$

\section{ACCESS}

Perhaps the largest hurdle facing patients with AD who may benefit from aducanumab is the annual price tag. As the AAN has pointed out in its public comments to ICER, patients with AD are likely to incur a significant amount of financial hardship given the recent trend in rising out-of-pocket costs for many neurologic medications. ${ }^{5}$ Traditionally, coverage determinations for Medicare are made without taking cost into consideration. For drugs covered under Part B, Medicare reimburses $106 \%$ of the average sales price (ASP). For drugs such as aducanumab, where an ASP is not available, Medicare pays $103 \%$ of the wholesale acquisition cost (WAC), which is currently $\$ 56,000$ per year for aducanumab. Based on an analysis by the Kaiser Foundation, nearly 2 million Medicare beneficiaries used 1 or more AD treatments covered under Part D in 2017. If even just a quarter of these patients are prescribed aducanumab $(\sim 500,000)$ at the reimbursement assumption of $103 \%$ of WAC costs, then the total spending of aducanumab in 1 year alone would be \$29 billion, which far exceeds spending on any other drug covered under Part B or Part D (for context, total Medicare spending for all Part B drugs was $\$ 37$ billion in 2019). Based on the current assumption for most Part B covered drugs and services for which Medicare pays $80 \%$ and beneficiaries are responsible for the remaining $20 \%$, beneficiaries would 
incur $\$ 11,500$ in coinsurance for 1 year of treatment with aducanumabnearly $40 \%$ of the $\$ 29,650$ median annual income of Medicare beneficiaries in 2019. ${ }^{12}$

Centers for Medicare \& Medicaid Services announced in July that it will conduct a National Coverage Determination (NCD) analysis for monoclonal antibodies directed against amyloids for the treatment of $\mathrm{AD}{ }^{13}$ The results of the NCD will ultimately determine how or if aducanumab is covered. The Alzheimer's Association has stated its support for the NCD, as well as a coverage with evidence development (CED). ${ }^{14}$

\section{Summary}

The lack of efficacy demonstrated in the clinical trial data coupled with the regulatory irregularities from the FDA have created a slippery slope for future approvals. The steep discount needed to reach the threshold price presents a conundrum for payers and patients with AD. The current approval of aducanumab may offer a glimmer of hope for a portion of patients with $\mathrm{AD}$, but it comes with a steep price tag that patients may not be able to afford. However, it must be noted that this is the first drug targeted for $\mathrm{AD}$ to be approved in almost 20 years and may provide some learnings to be carried forward in the quest for future viable treatments for patients with AD.

\section{DISCLOSURES}

No funding was received for the writing of this commentary. The author has nothing to disclose.

\section{REFERENCES}

1. Wong W. Economic burden of Alzheimer disease and managed care considerations. Am J Manag Care. 2020;26(8 Suppl): S177-S183.

2. Centers for Disease Control and Prevention. New estimates of Americans with Alzheimer's disease and related dementias show racial and ethnic disparities. September 20, 2018. Accessed August 18, 2021. https://www.cdc.gov/aging/ data/infographic/racial-ethnic-disparities-ad.html

3. Cavazzoni P. FDA's decision to approve new treatment for Alzheimer's disease. US Food and Drug Administration.

Updated June 7, 2021. Accessed August 18, 2021. https://www.fda.gov/ drugs/news-events-human-drugs / fdas-decision-approve-new-treatmentalzheimers-disease

4. Aduhelm (aducanumab-avwa). Prescribing information. Biogen Inc.; 2021. Accessed August 18, 2021. https://www. accessdata.fda.gov/drugsatfda docs/ label/2021/761178s000lbl.pdf

5. Lin GA WM, Synnott PG, et al. Aducanumab for Alzheimer's disease: effectiveness and value. Final evidence report and meeting summary. Institute for Clinical and Economic Review. August 5, 2021. Accessed October 4, 2021. https:// icer.org/wp-content/uploads/2020/10/ ICER_ALZ_Final_Report_080521.pdf

6. Institute for Clinical and Economic Review. Aducanumab for Alzheimer's disease: final policy recommendations. August 5, 2021. Accessed August 20, 2021. https://icer.org/wp-content/ uploads/2020/10/ICER ALZ Policy Recommendations_080521.pdf

7. Morris JC, Schindler SE, McCue LM, et al. Assessment of racial disparities in biomarkers for Alzheimer disease. JAMA Neurol. 2019;76(3):264-73.

8. Alzheimer's Association. Alzheimer's Association welcomes revised label for Aduhelm. Press release. July 8, 2021. Accessed September 7, 2021. https:// www.alz.org/news/2021/fda-reviseslabel-for-aduhelm-aducanumab
9. The Neurology Center. New Alzheimer's drug - aducanumab (Aduhel). Accessed September 27, 2021. https://www. neurologycenter.com/new-alzheimer-sdrug-aducanumab-aduhelm

10. Cleveland Clinic. Aducanumab FAQ. Accessed September 27, 2021. https:// my.clevelandclinic.org/departments/ neurological/depts/brain-health/ aducanumab-fag

11. Belluck P. Cleveland Clinic and Mount Sinai won't administer Aduhelm to patients. The New York Times. July 14, 2021. Updated September 2, 2021. Accessed September 27, 2021. https:// www.nytimes.com/2021/07/14/health/ cleveland-clinic-aduhelm.html

12. Cubanski J, Neumann T. FDA's approval of Biogen's new Alzheimer's drug has huge cost implications for Medicare and beneficiaries. Kaiser Family Foundation. June 10, 2021. Accessed September 27, 2021. https:// www.kff.org/medicare/issue-brief/ fdas-approval-of-biogens-new-alzheimers-drug-has-huge-cost-implications-formedicare-and-beneficiaries/

13. Centers for Medicare \& Medicaid Services. Medicare Coverage Center: national coverage determination analysis for monoclonal antibodies directed against amyloid for the treament of Alzheimer's disease. Accessed September 14, 2021. https://www.cms.gov/Center/ Special-Topic/Medicare-CoverageCenter\#skipNavTarget

14. Alzheimer's Association. Alzheimer's Association welcomes CMS announcement of national coverage determination analysis on treatment for Alzheimer's disease. Press release. July 12, 2021. Accessed September 14, 2021. https://www.alz. org/news/2021/alzheimers-associationwelcomes-cms-announcement-o 